\title{
Increased Nicotinamide Phosphoribosyltransferase and Cystathionine- $\beta$-Synthase in Renal Oncocytomas, Renal Urothelial Carcinoma, and Renal Clear Cell Carcinoma
}

\author{
RODNEY E. SHACKELFORD ${ }^{1}$, JEHAN ABDULSATTAR ${ }^{1}$, ERIC X. WEI ${ }^{1}$, \\ JAMES COTELINGAM ${ }^{1}$, DOMENICO COPPOLA ${ }^{2}$ and GUILLERMO A. HERRERA ${ }^{1}$ \\ ${ }^{1}$ Department of Pathology and Translational Pathobiology, LSU Health Shreveport, Shreveport, LA, U.S.A.; \\ ${ }^{2}$ Department of Anatomic Pathology, H. Lee Moffitt Cancer Center and Research Institute, Tampa, FL, U.S.A.
}

\begin{abstract}
Background: Renal oncocytomas (ROs), and clear cell (RCC) and urothelial carcinomas $(U C)$, are common renal neoplasms. Nicotinamide phosphoribosyltransferase (Nampt) catalyzes the rate-limiting step of $\mathrm{NAD}^{+}$synthesis and its expression is increased in several tumors. Nampt concomitantly regulates hydrogen sulfide $\left(\mathrm{H}_{2} \mathrm{~S}\right)$-synthesizing enzyme levels, including cystathionine- $\beta$-synthase (CBS). Materials and Methods: We used tissue microarrays to examine Nampt and the $\mathrm{H}_{2} \mathrm{~S}$-synthesizing enzyme CBS protein levels in benign kidney, RCC, UC and ROs. Results: Compared to benign kidney, all three neoplasms showed increased Nampt and CBS protein levels, with the levels increasing in RCC at higher Fuhrman grades. Conclusion: $\mathrm{H}_{2} \mathrm{~S}$ is known to ameliorate chronic renal failure but, as yet, no role for $\mathrm{H}_{2} \mathrm{~S}$ in renal neoplasia has been demonstrated. Here, we showed, for the first time, that Nampt, $\mathrm{CBS}$ and, likely, $\mathrm{H}_{2} \mathrm{~S}$ likely play a role in malignant and benign neoplastic renal disease.
\end{abstract}

Renal malignancies account for roughly $2.4 \%$ of adult malignancies, with renal clear cell carcinoma (RCC) being the most frequent renal parenchyma malignancy (70-85\%) and renal urothelial carcinoma (UC; 10-15\%) being the most common renal pelvic tumor $(1,2)$. Renal oncocytomas (ROs) are the most common benign renal neoplasms, accounting for $3-7 \%$ of renal neoplasms (1-3). RCC arises from the proximal

This article is freely accessible online.

Correspondence to: Rodney Shackelford, LSU Health Shreveport, Department of Pathology 1501 Kings HWY, Shreveport, LA 71130, U.S.A. Tel: +1 3186751228, Fax: +1 3186758395, e-mail: rshack@1suhsc.edu

Key Words: Renal cancer, nicotinamide phosphoribosyltransferase, Fuhrman grade, cystathionine- $\beta$-synthase. convoluted tubular epithelium and histologically consists of sheets and nests of cells with clear cytoplasm, containing lipid and glycogen, which have distinct cell boundaries in alveolar or tubulocystic arrangements (2). The Fuhrman classification system stratifies RCCs into four grades depending on nuclear size and shape, and nucleolar prominence at 100X and 400X magnifications (2). Renal UC arises in the renal pelvis and consists of nests and clusters of malignant epithelial cells histologically identical to bladder UC (4). Last, ROs are composed of cells with abundant eosinophilic granular cytoplasm and uniform small round, central nuclei with evenly dispersed chromatin that grow in nests and sheets in an often loose fibrous or myxoid stroma (5). ROs are immunoreactive to anti-mitochondrial antibodies and electron microscopy studies reveal numerous large cytoplasmic mitochondria (5).

At the molecular level, RCC frequently carries von HippelLindau tumor-suppressor gene mutations or loss, which causes uncontrolled hypoxia-inducible factor activation, leading to increased vascular endothelial growth factor, platelet-derived growth factor receptor, transforming growth factor- $\beta$ and erythropoietin expression. These in turn activate downstream targets, such as mTOR, ERK, and AKT, leading to increased angiogenesis, glycolysis, and suppressed apoptosis (2). UC often carries $T S C 1, p R B$, and $p 53$ inactivating mutations, combined with mTOR, PIK3CA, PI3K, and HRAS activation, leading to increased cell growth, mitosis, and angiogenesis with concomitant cell cycle checkpoint loss (6). Molecular analyses of ROs have shown that they fall into two categories; one diploid with CCND1 rearrangements/amplifications and an aneuploid type that carries recurrent chromosome $1, \mathrm{X}$ or $\mathrm{Y}$, and/or 14 and 21 losses $(3,7)$. This later type may progress to eosinophilic chromophobe renal cell carcinoma (7). Interestingly, most ROs show wild-type $p 53$, which likely acts as a barrier to malignant transformation and also causes slow growth and benign characteristics (7). 
Table I. Relative Nampt and CBS IHC staining in the tissue microarrays comparing benign renal tissue to renal UC, RO and RCC.

\begin{tabular}{|c|c|c|c|c|c|c|}
\hline \multirow{2}{*}{$\begin{array}{l}\text { Tissue Type } \\
\text { Antibody }\end{array}$} & \multicolumn{2}{|c|}{ Sample number } & \multicolumn{2}{|c|}{ Average IHC score } & \multicolumn{2}{|c|}{ SEM } \\
\hline & Nampt & CBS & Nampt & CBS & Nampt & CBS \\
\hline Benign Kidney & 21 & 11 & 2.95 & 2.09 & 0.50 & 0.90 \\
\hline Renal UC & 49 & 53 & 7.96 & 5.60 & 0.25 & 0.31 \\
\hline RO & 6 & 6 & 7.0 & 3.50 & 0.63 & 0.50 \\
\hline RCC Fuhrman I & 44 & 51 & 3.27 & 2.04 & 0.13 & 0.40 \\
\hline RCC Fuhrman II & 28 & 27 & 5.18 & 2.67 & 0.32 & 0.30 \\
\hline RCC Fuhrman III & 13 & 14 & 6.54 & 3.54 & 0.54 & 0.49 \\
\hline RCC Fuhrman IV & 9 & 9 & 8.67 & 7.33 & 0.71 & 0.53 \\
\hline
\end{tabular}

Nampt, Nicotinamide phosphoribosyltransferase; CBS, cystathionine- $\beta$-synthase; UC, urothelial carcinoma; RO, renal oncocytoma; RCC, renal clear cell carcinoma; IHC, immunohistochemistry; SEM, standard error of the mean.

Hydrogen sulfide $\left(\mathrm{H}_{2} \mathrm{~S}\right)$ plays a significant role in human malignancies and promotes cell-cycle progression, angiogenesis, changes in mitochondrial bioenergetics, and ablation of the cellular apoptotic responses (8). $\mathrm{H}_{2} \mathrm{~S}$ is synthesized by three enzymes; cystathionine- $\gamma$-lyase (CSE), 3 mercaptopyruvate sulfurtransferase (3-MST) and cystathionine$\beta$-synthase (CBS, 8). All three enzymes are increased in different cancer types (8-14). Increased CSE mRNA and protein are detected in human colon cancer HCT116 cells and its knockdown or inhibition in SW480 human colon cancer cell lines inhibits cell proliferation and migration (8-11). 3-MST is highly expressed in melanoma cell lines, but not in human colon cancer cells $(10,12)$. CBS and $\mathrm{H}_{2} \mathrm{~S}$ are increased in colon cancer cells compared to benign colonic epithelial cells, breast cancer-derived cells and ovarian cancer cells $(10,13$, 14). CBS also shows a concomitant increase with nicotinamide phosphoribosyltransferase (Nampt) in oral squamous cell carcinoma (15). Nampt catalyses the rate-limiting step of nicotinamide adenine dinucleotide synthesis and is increased in many types of cancer (16). Cellular $\mathrm{H}_{2} \mathrm{~S}$ concentrations, as well as CBS and Nampt protein levels form a positive feedback loop, with increased Nampt increasing $\mathrm{H}_{2} \mathrm{~S}$ concentrations and CBS protein expression, with both increasing cancer cell survival, proliferation, and dedifferentiation (16-18). Last, $59.5 \%$ of RCCs, $26.7 \%$ of ROs, and $85.7 \%$ of UCs are immunoreactive for enzymatically active phospho-Stat3 (19). Stat3 activation induces Nampt protein expression, thus, the three neoplasms examined here are likely to carry a signal transduction pathway that induces Nampt and CBS protein expression $(17,20)$.

In the kidney, $\mathrm{H}_{2} \mathrm{~S}$ regulates excretory functions, blood pressure through its effects on juxtaglomerular cells and exerts anti-inflammatory, anti-oxidant, and anti-apoptotic effects (21). Presently, no role for $\mathrm{H}_{2} \mathrm{~S}$ in renal neoplasia has been identified. Based on the current data concerning the roles of Nampt, $\mathrm{H}_{2} \mathrm{~S}$, and the $\mathrm{H}_{2} \mathrm{~S}$-synthsizing enzymes in human malignancy, we used tissue microarray to examine Nampt and CBS protein levels in benign human kidney, RO, $\mathrm{RCC}$, and renal UC.

\section{Materials and Methods}

Upon Institutional Review Board approval by LSU Health Shreveport, two tissue microarrays (TMAs; catalog number KD208) were purchased from US Biomax, Inc. (Rockville, MD, USA). The TMAs contained 29 benign renal cortical tissue samples, 57 renal UCs, 6 ROs and 107 RCCs. The RCC Fuhrman grades were grade I 54 samples, grade II 29 samples, grade III 15 samples and grade IV 9 samples. All tissue samples in the TMAs were $1.0 \mathrm{~mm}$ in diameter.

Nampt and CBS immunohistochemistry (IHC). The concentration of primary Nampt and CBS antibodies were optimized to normal kidney as control tissue. The staining of the TMAs was performed in the Tissue Core Histology Lab Facility at the Moffitt Cancer Center (Tampa, FL, USA). The microarray slides were stained using a Ventana Discovery XT automated system (Ventana Medical Systems, Tucson, AZ, USA) as per the manufacturer's protocol with proprietary reagents. Briefly, the slides were deparaffinized on the automated system with EZ Prep solution (\#950-100; Ventana Medical Systems). The heat-induced antigen retrieval method was used in Cell Conditioning 1 (\#950-124; Ventana Medical Systems). Mouse monoclonal antibody to human Nampt (\#ALX-804-717; Enzo life Sciences, Plymouth Meeting, PA, USA) was used at a 1:1000 concentration in Dako antibody diluent (\#S0809; Dako, Carpenteria, CA, USA) and incubated for $60 \mathrm{~min}$. The Abcam mouse monoclonal antibody to human CBS (ab54883; Cambridge, MA, USA) was used under the same conditions. The Ventana antimouse secondary antibodies were used for $16 \mathrm{~min}$. The detection system used was the Ventana OmniMap kit. Slides were then dehydrated and cover-slipped as per standard laboratory protocol.

Evaluation of Nampt and CBS staining. Relative Nampt and CBS protein expression was determined as immunostain intensity scored on a $0-3$ scale as follows: no staining as 0 , light staining as 1 , moderate staining as 2 and heavy staining as 3 . The percentage of cells stained was measured, with no detectable staining as $0,1-33 \%$ 


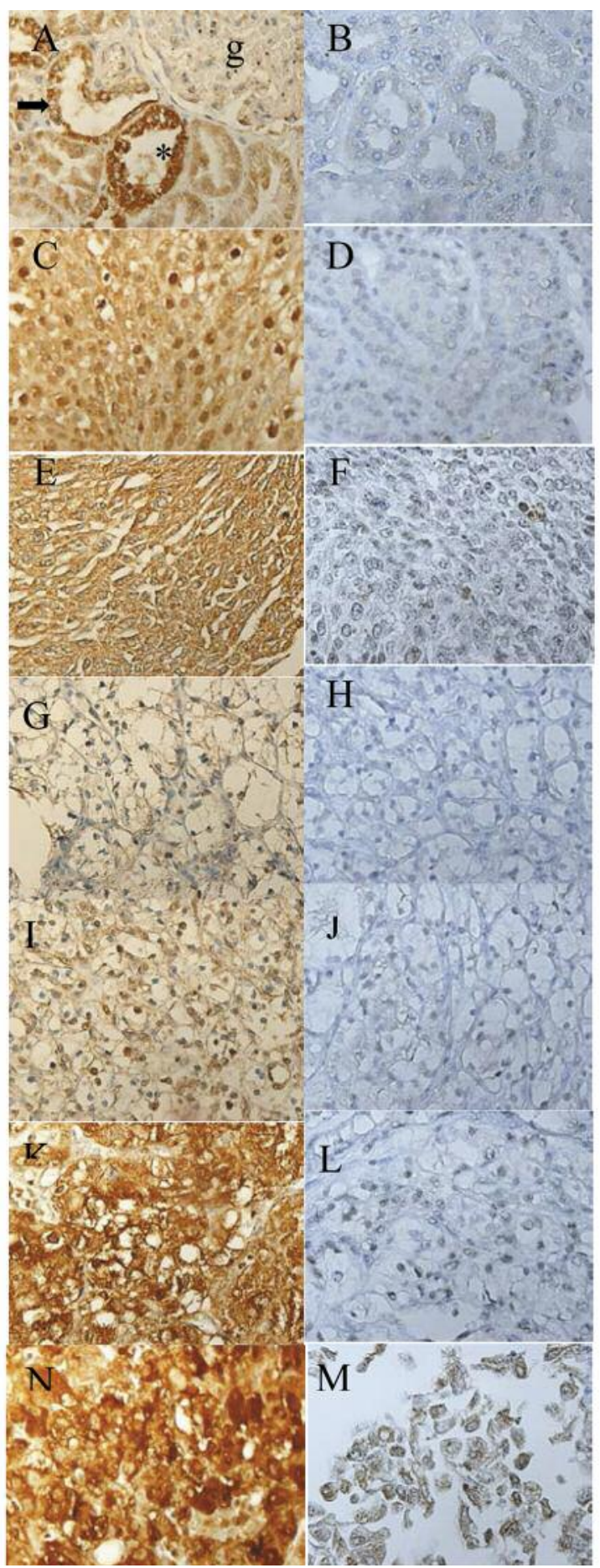

Figure 1. Representative high-power views of Nampt and CBS IHC. Benign renal cortex Nampt $(A)$ and $C B S(B)$. “*” denotes the proximal renal tubule, the arrow the distal renal tubule and " $g$ " the glomerulus. $R O$ Nampt $(C)$ and CBS $(D)$, renal UC Nampt $(E)$ and CBS $(F)$, Fuhrman grade I Nampt $(G)$ and CBS $(H)$, Fuhrman grade II Nampt (I) and CBS (J), Fuhrman grade III Nampt $(K)$ and CBS $(L)$, Fuhrman grade IV Nampt $(M)$ and $C B S(N)$. $G$ through $N$ are RCC. Nampt, Nicotinamide phosphoribosyltransferase; $C B S$, cystathionine- $\beta$-synthase; UC, urothelial carcinoma; RO, renal oncocytoma; IHC, immunohistochemistry. as $1,34-66 \%$ as 2 and $67-100 \%$ as 3 . The final IHC score was the product of the percentage of cells stained multiplied by the intensity score, allowing for a maximal score of 9 and a minimal score of 0 . Nuclear and cytoplasmic Nampt and CBS staining were seen in all tissue samples examined, although at low levels in benign kidney and at very low levels in Fuhrman grade I RCC due to the cytoplasmic clearing. We, therefore, measured and quantified Nampt and CBS staining in the nuclear and cytoplasmic compartments.

Statistical analysis. The standard error of the mean (SEM) IHC score was calculated by taking the mean of each data set, subtracting the mean from each number in the set and squaring the result, then calculating the mean of the squared differences and taking the square root of this number to find the standard deviation. The standard deviation was then divided by the square root of the number of tissue samples in the sample set to find the SEM.

\section{Results}

Following IHC processing, we were left with 21 benign renal cortex samples, 49 UC samples, 6 RO samples and 94 RCC samples (44 Fuhrman grade I, 28 grade II, 13 grade III and 9 grade IV) for the TMA probed with the anti-Nampt antibody. For the TMA probed with the anti-CBS antibody, we were left with 11 benign renal cortex samples, 53 UC samples, 6 RO sample and 101 RCC samples (51 Fuhrman grade I, 27 grade II, 14 grade III and 9 grade IV). Some cases were lost in IHC processing; hence, fewer cases were analyzed than were on the TMAs. The number of cases examined with each antibody, the quantified IHC results and the SEM of each data set are given in Table I. Examples of Nampt and CBS IHC of sample tissues are shown in Figure 1.

\section{Discussion}

Nampt is highly expressed in several human malignancies, including thyroid, colorectal, gastric, prostate, endometrial, and ovarian cancers (16). Here, we show, for the first time, that Nampt and CBS protein levels are increased in RCC, renal UC, and ROs compared to benign renal cortex. Nampt expression in the renal cortex was highest in the proximal convoluted tubules, intermediate in the distal convoluted tubules, and low in the glomerulus (Figure 1). Nampt expression was high in renal UC and in RCC increased at higher Fuhrman grades. Interestingly, Nampt was also relatively high in the $\mathrm{RO}$, indicating that it can show increased expression in benign renal neoplasms. CBS was expressed at very low levels in benign renal cortex, predominately in the cytoplasm. In the renal UC, CBS was moderately increased in the nucleus and cytoplasm and only slightly increased in the ROs. In RCC Fuhrman grade I, CBS showed weak nuclear and cytoplasmic staining, slightly below that of benign renal cortex. CBS immunostaining increased with increasing Fuhrman grades and was most highly expressed in Fuhrman grade IV RCC. 
Previously, Nampt and CBS protein levels were examined in oral squamous cell carcinoma by TMA analysis (15). Both Nampt and CBS were increased in oral squamous cell carcinoma compared to benign oral epithelium. Interestingly, both proteins showed increased expression with higher tumor grades, with Nampt levels being higher at each grade than were CBS levels (15). Similarly, in a TMA study of rhabdomyosarcomas and leiomyosarcomas, Nampt levels increased with increased leiomyosarcoma grade and positively correlated with rhabdomyosarcoma-subtype clinical aggressiveness (22). These data, combined with our findings here, support the hypothesis that Nampt and CBS increase with increasing tumor grade. Additionally, we show that Nampt and CBS are increased in the RO, indicating both enzymes are increased in benign renal neoplasia. Fuhrman grade I RCC cytoplasm usually has a high lipid/glycogen content with concomitant lower metabolic activity, while higher grade RCCs often show increasingly granular/ eosinophilic cytoplasm with increased metabolic activity (2, $5,23,24)$. Increasing Nampt and CBS at higher Fuhrman grades are likely an important component of these changes. CBS was expressed at low levels in low-grade oral squamous cell carcinoma (15). Its low expression in Fuhrman grade I RCC is likely due to this enzyme being expressed at low levels in benign tissue and lower-grade malignant tumors, as was previously found in oral squamous cell carcinomas (Table I, Figure 1) (15).

Here, we show, for the first time, that Nampt, CBS and likely $\mathrm{H}_{2} \mathrm{~S}$ are increased in RCC, RO, renal UC. Although preliminary, our findings likely indicate that $\mathrm{H}_{2} \mathrm{~S}$ plays an important role in benign and malignant renal neoplastic disease. Further research, particularly on renal cancer's $\mathrm{H}_{2} \mathrm{~S}$ concentrations and possible dysregulation of 3-MST and CSE expression, are underway.

\section{Acknowledgements}

The Authors thank the Histology Section of the Tissue Core at the Moffitt Cancer Center and Research Institute for the support in performing the IHC stains.

\section{References}

1 Ferlay J, Soerjomataram I, Dikshit R, Eser S, Mathers C, Rebelo M, Parkin DM, Forman D and Bray F: Cancer incidence and mortality worldwide: Sources, methods and major patterns in GLOBOCAN 2012. Int J Cancer 136: 359-386, 2015.

2 Hsieh JJ, Purdue MP, Signoretti S, Swanton C, Albiges L, Schmidinger M, Heng DY, Larkin J and Ficarra V: Renal cell carcinoma. Nat Rev Dis Primers 3: 17009, 2017.

3 Trpkov K, Yilmaz A, Uzer D, Dishongh KM, Quick CM, Bismar $\mathrm{TA}$ and Gokden N: Renal oncocytoma revisited: A clinicopathological study of 109 cases with emphasis on problematic diagnostic features. Histopathology 57: 893-906, 2010.
4 Gupta R, Paner GP and Amin MB: Neoplasms of the upper urinary tract: A review with focus on urothelial carcinoma of the pelvicalyceal system and aspects related to its diagnosis and reporting. Adv Anat Pathol 15: 127-139, 2008.

5 Morra MN and Das S: Renal oncocytoma: A review of histogenesis, histopathology, diagnosis and treatment. J Urol 150: 295-302, 1993 .

6 Solomon JP and Hansel DE: Morphologic and molecular characteristics of bladder cancer. Surg Pathol Clin 8: 663-676, 2015.

7 Joshi S, Tolkunov D, Aviv H, Hakimi AA, Yao M, Hsieh JJ, Ganesan S, Chan CS and White E: The genomic landscape of renal oncocytoma identifies a metabolic barrier to tumorigenesis. Cell Rep 13: 1895-908, 2015.

8 Wu D, Si W, Wang M, Lv S, Ji A and Li Y: Hydrogen sulfide in cancer: Friend or foe? Nitric Oxide 50: 38-45, 2015.

9 Fan K, Li N, Qi J, Yin P, Zhao C, Wang L, Li Z and Zha X: $\mathrm{Wnt} / \beta$-catenin signaling induces the transcription of cystathionine- $\gamma$-lyase, a stimulator of tumor in colon cancer. Cell Signal 26: 2801-2808, 2014.

10 Szabo C, Coletta C, Chao C, Modis K, Szczesny B, Papapetropoulos A and Hellmich MR: Tumor-derived hydrogen sulfide, produced by cystathionine- $\beta$-synthase, stimulates bioenergetics, cell proliferation, and angiogenesis in colon cancer. Proc Natl Acad Sci USA 110: 12474-12479, 2013.

11 Cai WJ, Wang MJ, Ju LH, Wang C and Zhu YC: Hydrogen sulfide induces human colon cancer cell proliferation: Role of Akt, ERK and p21. Cell Biol Int 34: 565-572, 2010.

12 Germano D, Napolitano M, Papapetropoulos A, Bucci M, Cirino $\mathrm{G}$ and Ianaro A: Role of the cystathionine- $\gamma$-lyase/hydrogen sulfide pathway in human melanoma progression. Pigment Cell Melanoma Res 28: 61-72, 2015.

13 Bhattacharyya S, Saha S, Giri K, Lanza IR, Nair, KS, Jennings NB, Rodriguez-Aguayo C, Lopez-Berestein G, Basal E, Weaver AL, Visscher DW, Cliby W, Sood AK, Bhattacharya B and Mukherjee P: Cystathionine- $\beta$-synthase (CBS) contributes to advanced ovarian cancer progression and drug resistance. PLoS One 8: e79167, 2013.

14 Sen S, Kawahara B, Gupta D, Tsai R, Khachatryan M, Roy Chowdhuri S. Bose S, Yoon A, Faull K, Farias-Eisner R and Chaudhuri G: Role of cystathionine- $\beta$-synthase in human breast cancer. Free Radic Biol Med 86: 228-238, 2015.

15 Patel S, Ansari J, Meram A, Abdulsattar J, Cotelingam J, Coppola D, Ghali G and Shackelford R: Increased nicotinamide phosphoribosyltransferase and cystathionine-beta-synthase in oral cavity squamous cell carcinomas. Int J Clin Exp Pathol 10: 702-707, 2017.

16 Shackelford RE, Mayhall K, Maxwell NM, Kandil E and Coppola D: Nicotinamide phosphoribosyltransferase in malignancy: A review. Genes Cancer 4: 447-56, 2013.

17 Sanokawa-Akakura R, Ostrakhovitch EA, Akakura S, Goodwin $\mathrm{S}$ and Tabibzadeh S: $\mathrm{A}_{2} \mathrm{~S}$-Nampt dependent energetic circuit is critical to survival and cytoprotection from damage in cancer cells. PLoS One 9: e108537, 2014.

18 Ostrakhovitch EA, Akakura S, Sanokawa-Aka-kura R, Goodwin $\mathrm{S}$ and Tabibzadeh S: Dedifferentiation of cancer cells following recovery from a potentially lethal damage is mediated by $\mathrm{H}_{2} \mathrm{~S}$ Nampt. Exp Cell Res 330: 135-150, 2015.

19 Guo C, Yang G, Khun K, Kong X, Levy D, Lee P and Melamed $\mathrm{J}$ : Activation of Stat3 in renal tumors. Am J Transl Res 1: 283290, 2009. 
20 Nowell MA, Richards PJ, Fielding CA, Ognjano-vic S, Topley $\mathrm{N}$, Williams AS, Bryant-Greenwood G and Jones SA: Regulation of pre-B cell colony-enhancing factor by STAT-3-dependent inter-leukin-6 trans-signaling: implications in the pathogenesis of rheumatoid arthritis. Arthritis Rheum 54: 2084-2095, 2006.

$21 \mathrm{Cao} X$ and Bian JS: The role of hydrogen sulfide in renal system. Front Pharmacol 7: 385, 2016.

22 Vora M, Ansari J, Shanti RM, Veillon D, Cotelingam C, Coppola $\mathrm{D}$ and Shackelford RE: Increased nicotinamide phosphoribosyltransferase in rhabdomyosarcomas and leiomyosarcomas compared to skeletal and smooth muscle tissue. Anticancer Res 36: 503-508, 2016.
23 López JI: Renal tumors with clear cells. A review. Pathol Res Pract 209: 137-146, 2013.

24 Dong B, Gao Y, Kang X, Gao H, Zhang J, Guo H, You MJ, Xue $\mathrm{W}$, Cheng $\mathrm{J}$ and Huang Y: SENP1 promotes proliferation of clear cell renal cell carcinoma through activation of glycolysis. Oncotarget 7: 80435-80449, 2016.
Received May 9, 2017

Revised May 22, 2017

Accepted May 24, 2017 